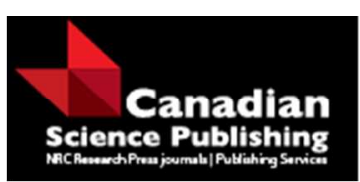

Canadian Journal of Forest Research

Revue canadienne de recherche forestière

\title{
VORONOI POLYGONS QUANTIFY BIAS WHEN SAMPLING THE NEAREST PLANT
}

\begin{tabular}{|r|l|}
\hline Journal: & Canadian Journal of Forest Research \\
\hline Manuscript ID & cjfr-2015-0238.R1 \\
\hline Manuscript Type: & Note \\
\hline Date Submitted by the Author: & 01-Aug-2015 \\
\hline Complete List of Authors: & Lynch, Thomas; Oklahoma State University, \\
\hline Keyword: & $\begin{array}{l}\text { increment cores, distance sampling, k tree sampling, Area potentially } \\
\text { available, Forest sampling }\end{array}$ \\
\hline \multicolumn{2}{|l}{} \\
\hline
\end{tabular}

SCHOLARONE $^{\text {M }}$
Manuscripts 
1

2

3

4

5

6

7

8

9

10

11

12

13

14

15

16
Thomas B. Lynch

Professor

Department of Natural Resource Ecology and Management

Oklahoma State University

Stillwater, OK 74078

Email: tom.1ynch@,okstate.edu

Phone: 405-477-5447

FAX: 405-744-3530

Corresponding author - Thomas B. Lynch with contact information given above

https://mc06.manuscriptcentral.com/cjfr-pubs 

VORONOI POLYGONS QUANTIFY BIAS WHEN SAMPLING THE NEAREST PLANT ABSTRACT: The design-bias in the sample mean obtained from sampling the trees nearest to points randomly and uniformly distributed over a forested area can be exactly quantified in terms of the Voronoi polygons (V-polygons) surrounding each tree in the forest of interest. For this sampling method the V-polygon for a prospective sample tree is its inclusion zone. The sides of such polygons are perpendicular to a line joining adjacent trees and equidistant from these trees.

24 For any individual tree attribute $Y$, the design-bias in such a sample mean for estimating the 25 population mean of $Y$ will be equal to the covariance between $Y$ and V-polygon area $V$ divided by the mean $\mathrm{V}$-polygon area. The bias as a percent of the population mean of $Y$ is the product of

27 the correlation coefficient between $Y$ and $V$ and the coefficients of variation for $Y$ and $V$

28 multiplied by 100. This implies that attempts to estimate the means of commonly-measured

29 individual tree variables such as $\mathrm{DBH}$, basal area, and crown diameter or area from sampling 30 trees nearest to randomly-located points will likely be positively biased and the magnitude of that 31 bias will depend on the strength of the linear relationship to V-polygon area as well as the 32 variability among the $\mathrm{V}$-polygon areas and the variable of interest. It is not obvious whether 33 increment core data will be positively or negatively biased because this depends on the 34 characteristics of the forest of interest. The main conclusion of the study is that the bias formula 35 derived for unweighted estimation from sampling the tree nearest to a point indicates that bias in 36 the range of $5-10 \%$ or greater can occur in many forest populations.

37 KEY WORDS: increment cores, distance sampling, $k$-tree sampling, APA, Area Potentially 38 Available, inclusion zone, forest sampling, forest inventory 


\section{INTRODUCTION}

42 Although ecologists and foresters have sometimes used samples obtained from selecting the 43 nearest tree or other plant to a point or plot center in the field to estimate population means for

44 per-tree attributes such as growth from increment cores, Clutter et al. (1983, p. 119) pointed out 45 that this procedure is more likely to sample "relatively open-grown" trees possibly resulting in 46 biased estimators. These remarks were made in a discussion of growth predictions obtained

47 from the "stand table projection" procedure because increment core samples for the procedure 48 had often been obtained by sampling the tree nearest to a sample point or plot center in the field.

49 However, Clutter et al. (1983, p.119) did not provide an exact formula for the magnitude of this

50 bias. Clutter et al. (1983, p. 119) recommended that increment cores be obtained by sampling all

51 trees on small fixed-radius plots.

52 Barabesi and Fattorini (1998) point out that Overton and Stehman (1995) have suggested a

53 design-based procedure for estimating attributes of plants nearest to randomly points. In their

54 method the probability of selection is proportional to the area of the V-polygon (Voronoi

55 polygon, Wikipedia authors 2015) surrounding the plant, allowing a Horvitz-Thompson

56 estimator to be used. V-polygons can be constructed by employing perpendicular bisectors

57 between adjacent plants. Figure 1 illustrates a typical Voronoi tessellation for a synthetic forest 58 population developed using a spatial inhibition process that had $3 \mathrm{~m}$ inhibition distance (Venables 59 and Ripley, 2002, p. 434). The package "spatial” was used in R (R Development Core Team, 60 2012). Figure 1 was then produced by using the "deldir" package (Turner, 2015) in R. The V- 
61 polygon associated with a prospective sample tree is its inclusion zone for sampling because

62 whenever a randomly-located point falls in this V-polygon (inclusion zone), it will be closer to

63 that tree than any other in the population and hence will be selected as a sample tree. However

64 Barabesi and Fattorini (1998) conclude that the construction of V-polygons in the field would be

65 "very impractical and time consuming." Kleinn and Vilkĉo (2006) give a thorough review of the

66 application of V-polygons to ecological sampling and extend the theory of design-based V-

67 polygon sampling to the situation in which the nearest $k$ trees to a random point are selected and

68 higher-order V-polygons are employed. However, they conclude that the practicality of

69 employing these ideas in the field with current technology is probably limited due to the

70 measurements required to construct higher-order V-polygons. Related sampling concepts that

71 are intended to reduce sampling effort in the field were triangulation methods proposed by

72 Fehrmann et al. (2011). Foresters have long used the concept of the Veronoi polygon under the

73 title of APA (Area Potentially Available, Mercier and Baujard (1997), Brown (1965), Mead

74 (1966), Avery and Burkhart 2002 p. 331-332). APA has been used in the development of forest

75 growth models.

76 Consider a forest tract having area $A$ tessellated into $\mathrm{V}$-polygons for each tree such that when a

77 randomly located point in area $A$ falls into the $\mathrm{V}$-polygon of area $V_{i}$ associated with tree $i$ then

78 tree $i$ will be selected as a sample tree. In the latter case tree $i$ will be the nearest tree to the

79 randomly located point in area $A$. We want to sample attribute $Y_{i}$ (e.g., increment core data,

80 basal area, diameter at breast height $(\mathrm{DBH}))$ which is an individual tree characteristic associated

81 with tree $i$ within a V-polygon associated with that tree. It is clear that for this procedure the

82 probability of selecting $Y_{i}$ on a V-polygon having area $V_{i}$ will be $V_{i} / A$. It should be noted that for

83 trees or other plants near the edge of the tract (which could be the tract boundary, pond, etc.) part 
84 of the V-polygon border may include tract edge, and this may not be half the distance to a 85 nearest neighboring plant.

86 Lappi and Bailey (1987) investigated a very similar situation in which increment cores were

87 selected on angle-count (horizontal point or Bitterlich) sampling trees. They found that the bias

88 in estimating the mean of a variable $y$ on trees selected in a point sample was equal to the

89 covariance between $\mathrm{DBH}^{2}$ and $y$ divided by the population mean value of $\mathrm{DBH}^{2}$. Since angle-

90 count sample trees are selected with probability proportional to $\mathrm{DBH}^{2}$ the situation is closely

91 analogous to sampling $Y_{i}$ on $\mathrm{V}$-polygons with probability proportional to $V_{i}$. Angle-count

92 sampling is based on probability proportional to size sampling while selection of the plant nearst

93 to a random point samples with probability proportional to the V-polygon of the selected tree.

94 Applying Lappi and Bailey's (1987) methods to the problem of sampling the nearest plant or tree 95 to a randomly-located point leads to a bias similar to that found by those researchers. The bias is 96 equal to the covariance between $V_{i}$ and $Y_{i}$ divided by the population mean area of the of $\mathrm{V}$ -

97 polygons $\mu_{V}$. This will be derived below using a somewhat different presentation based on the 98 work of Palley and Horwitz (1961) in point sampling.

99 We now want to consider the expected value of the average of the individual tree characteristic $100 Y_{i}$ associated with the $\mathrm{V}$-polygon of area $V_{i}$ based on a sample of $n$ points independently and 101 randomly located with a uniform distribution over the tract area $A$.

102 METHODS

103 We will be considering a finite population of trees with associated V-polygons from which a 104 random sample of size $n$ will be drawn. Sample trees will be selected when a randomly-located 105 point on the tract falls within the V-polygon area of a sample tree. Using this model we can 
106 calculate the expected value of the sample mean and quantify the bias in the sample mean

107 estimate of any tree attribute for estimation of the population mean of that attribute. We begin

108 by describing the population and some of its parameters below.

109 Preliminaries:

110 We have a finite population of $N \mathrm{~V}$-polygons ( one for each tree) each with area $V_{i}$ and so we can

111 define the first and second moments of this finite population as well as the variance as follows

112 (the first moment being the population mean):

$\mu_{V}=\frac{1}{N} \sum_{i=1}^{N} V_{i}$, the population mean for $V$,

$113 \mu_{2 V}^{\prime}=\frac{1}{N} \sum_{i=1}^{N} V_{i}^{2}$, the second moment for $V$, and

$\sigma_{V}^{2}=\mu_{2 V}^{\prime}-\mu_{V}^{2}$, the population variance for $V$.

114 And note that the land area $A$ is the sum of the V-polygon areas:

$115 \quad A=\sum_{i=1}^{N} V_{i}$

116 Noting that the area $V_{i}$ will be the area of the inclusion zone for tree $i$, we define a Bernoulli

117 random variable $Z_{i j}$ associated with random point location as follows:

$118 Z_{i j}=1$ if random point $j$ falls in the $\mathrm{V}$-polygon of area $V_{i}$, otherwise

$119 Z_{i j}=0$

120 We then clearly have the inclusion probability (probability of inclusion in the sample with

121 random point $i$ ) and the probability of not being in the sample:

122

$P\left(Z_{i j}=1\right)=\frac{V_{i}}{A}$ for all $j$, and

$P\left(Z_{i j}=0\right)=1-\frac{V_{i}}{A}$ for all $j$. 
123 We also have a finite population of $N$ individual tree attributes $Y_{i}$ each associated with tree $i$

124 located on a V-polygon of area $V_{i}$. Parameters associated with this population include:

$\mu_{Y}=\frac{1}{N} \sum_{i=1}^{N} Y_{i}$, the population mean for $Y$,

$125 \quad \mu_{2 Y}^{\prime}=\frac{1}{N} \sum_{i=1}^{N} Y_{i}^{2}$, the second moment for $Y$, and

$\sigma_{Y}^{2}=\mu_{2 Y}^{\prime}-\mu_{Y}^{2}$, the population variance for $Y$.

126 Parameters concerning the relationship between the individual tree attribute $Y_{i}$ and the area of the

127 V-polygon $V_{i}$ are:

$\mu_{Y V}=\frac{1}{N} \sum_{i=1}^{N} Y_{i} V_{i}$

$128 \sigma_{Y V}=\mu_{Y V}-\mu_{Y} \mu_{V}$, the population covariance for $Y$ and $V$, and

$\rho_{Y V}=\frac{\sigma_{Y V}}{\sigma_{Y} \sigma_{V}}$ the correlation coefficient between $Y$ and $V$.

The expected value of the sample mean of tree attribute $Y$ from point to nearest tree samples:

131 Following a formulation similar to that used by Palley and Horwitz (1961) for point sampling we

132 can then express the sample mean of $Y_{i}$ from $n$ independent randomly and uniformly located

133 points over tract area $A$, intended to be an estimator of $\mu_{Y}$, as:

$$
\bar{Y}_{R P}=\frac{1}{n} \sum_{j=1}^{n} \sum_{i=1}^{N} Y_{i} Z_{i j}
$$

134 where

$\bar{Y}_{R P}$ is the sample mean based on random point $(R P)$ samples

135 We now want to find the expected value of this estimator: 
$136 \quad E\left(\bar{Y}_{R P}\right)=E\left(\frac{1}{n} \sum_{j=1}^{n} \sum_{i=1}^{N} Y_{i} Z_{i j}\right)$

137 And by using the properties of expected values, since the $n$ random points are independently

138 identically distributed:

$$
\begin{aligned}
& E\left(\bar{Y}_{R P}\right)=E\left(\frac{1}{n} \sum_{j=1}^{n} \sum_{i=1}^{N} Y_{i} Z_{i j}\right) \\
139 \quad= & \frac{1}{n} \sum_{j=1}^{n} \sum_{i=1}^{N} Y_{i} E\left(Z_{i j}\right)=\frac{1}{n} n \sum_{i=1}^{N} Y_{i} E\left(Z_{i 1}\right) \\
= & \sum_{i=1}^{N} Y_{i} E\left(Z_{i 1}\right)
\end{aligned}
$$

140 It is well-known that the expected value of a Bernoulli random variable is equal to the

141 probability of its occurrence, so:

$$
\begin{aligned}
& E\left(\bar{Y}_{R P}\right)=\sum_{i=1}^{N} Y_{i} E\left(Z_{i 1}\right) \\
= & \sum_{i=1}^{N} Y_{i} \frac{V_{i}}{A} \\
142 \quad= & \frac{1}{A} \sum_{i=1}^{N} Y_{i} V_{i}
\end{aligned}
$$

and because $A=N \mu_{V}$ we also have :

$E\left(\bar{Y}_{R P}\right)=\frac{1}{N \mu_{V}} \sum_{i=1}^{N} Y_{i} V_{i}=\frac{\mu_{Y V}}{\mu_{V}}$

143 From the covariance between $Y$ and $V$ we have:

144

$\sigma_{Y V}=\mu_{Y V}-\mu_{Y} \mu_{V}$, the population covariance for $Y$ and $V$, and

$\Rightarrow \mu_{Y V}=\sigma_{Y V}+\mu_{Y} \mu_{V}$

145 Now combining (9) and (10) yields:

$146 E\left(\bar{Y}_{R P}\right)=\frac{\mu_{Y V}}{\mu_{V}}=\frac{\sigma_{Y V}+\mu_{Y} \mu_{V}}{\mu_{V}}=\mu_{Y}+\frac{\sigma_{Y V}}{\mu_{V}}$ 
147 Therefore, if we were trying to estimate the mean of $Y, \mu_{Y}$, by selecting sample trees nearest to a 148 randomly located (uniformly distributed over the tract of area $A$ ) point the design-bias is:

$$
\operatorname{Bias}_{\bar{Y}_{R P}}=\frac{\sigma_{Y V}}{\mu_{V}}=\frac{\rho_{Y V} \sigma_{Y} \sigma_{V}}{\mu_{V}}=\rho_{Y V} \sigma_{Y} C_{V}
$$

149 where

$$
C_{V}=\frac{\sigma_{V}}{\mu_{V}}, \text { the coefficient of variation for } V
$$

150 This is the result that we might have expected based on the work of Lappi and Bailey (1987) in

151 the context of point sampling. Indeed this is the bias in any case in which an unweighted

152 average of probability proportional to size samples is obtained, where $V$ would be replaced by

153 any other variable that is proportional to the probability of selection. Equation 11 has also been

154 presented by Gove and Patil (1998, Appendix B) as part of their study of weighted distributions

155 with applications to basal area distributions and as well by Patil and Rao (1978, Theorems $1 \&$ 2)

156 but in neither case was connected toV-polygons or distance sampling as presented here. The

157 Gove and Patil (1998, Appendix B) result is more general than Equation 11 in that it was derived 158 for any weighted distribution. The derivation of Equation 11 given here differs from Gove and 159 Patil (1998), Patil and Rao (1978) and Lappi and Bailey (1987) in that it is done in a design160 based framework using the approach of Palley and Horwitz (1961).

161 This design-bias as a percent of the population mean value of $Y, \mu_{Y}$, is then:

$$
\text { Bias } \%_{\bar{Y}_{R P}}=\frac{\rho_{Y V} \sigma_{Y} C_{V}}{\mu_{Y}} 100=\rho_{Y V} C_{Y} C_{V} 100
$$

162 where

$$
C_{Y}=\frac{\sigma_{Y}}{\mu_{Y}} \text {, the coefficient of variation for } V
$$


163 For example if the correlation between $Y$ and the V-polygon area $V$ was 0.8 and both $Y$ and $V$ had 164 correlation coefficients of $30 \%$ the bias from selecting sample trees nearest to a randomly 165 selected point would be $0.8 \times 0.3 \times 0.3 \times 100=7.2 \%$. Clearly the bias percent in equation (13) will 166 be positive when the correlation between $Y$ and the size of the $\mathrm{V}$-polygon $\mathrm{V}$ is positive but 167 negative when the correlation between $Y$ and the size of the $\mathrm{V}$-polygon $\mathrm{V}$ is negative. Zero 168 correlation would of course imply no bias. Low bias is also expected if the coefficient of 169 variation for $Y$ or $V$ or both is low. Because a tree $\mathrm{V}$-polygon is equivalent to its APA as used in 170 forest growth simulators (e.g. Burkhart and Avery 2002 p. 331-332) we would often expect 171 correlations between $\mathrm{V}$-polygon size and variables such as $\mathrm{DBH}$, individual tree basal area and 172 crown area to be positive leading to a positive bias for these variables.

173 The expected size of the V-polygon for sampling the tree nearest to a random point

174 An interesting special case of estimator 6 occurs if we want to estimate the average size of the V175 polygon associated with choosing the tree nearest to a random (uniformly distributed) point in 176 the forest of interest. We then have:

$177 \quad \bar{V}_{R P}=\frac{1}{n} \sum_{j=1}^{n} \sum_{i=1}^{N} V_{i} Z_{i j}$

178 Because the covariance of a variable with itself is identical to the variance for that variable, from 179 equation 12 the bias in estimating $\mu_{V}$ from this procedure is:

$180 \operatorname{Bias}_{\bar{V}_{R P}}=\frac{\sigma_{V}^{2}}{\mu_{V}}=\mu_{V} C_{V}^{2}$ 
181 Equation 15 is equivalent to Patil and Rao (1978) Theorem 1 which they derived for general

182 weighted distributions. As indicated above, they did not connect their result to V-polygons as is 183 done here. And from equation 13 the bias as a percentage of $\mu_{V}$ is then:

$184 \quad$ Bias $\%_{\bar{V}_{R P}}=\frac{\sigma_{V} C_{V}}{\mu_{V}} 100=C_{V}^{2} 100$

185 For example a forest for which the coefficient of variation among V-polygons was 30\% would

186 have a bias of $9 \%$ for $\bar{V}_{R P}$. Naturally this bias would always be greater than or equal to zero.

187 Weighted estimation

188 If measurements of the areas of the V-polygons $V_{i}$ were feasible, the following per-tree estimator 189 which samples are weighted by the inverse probability of selection for tree $i, V_{i} / A$ could be used 190 to estimate the population mean of $Y, \mu_{Y}$ :

191

$$
\bar{Y}_{R P}^{w}=\frac{\sum_{j=1}^{n} \sum_{i=1}^{N} \frac{A Y_{i}}{V_{i}} Z_{i j}}{\sum_{j=1}^{n} \sum_{i=1}^{N} \frac{A}{V_{i}} Z_{i j}}
$$

$\bar{Y}_{R P}^{w}$ is the weighted estimator for the mean of $Y, \mu_{Y}$

192 This is a ratio of means estimator in which the numerator and denominator are design-unbiased

193 estimates of the sum of tree characteristics $Y_{i}$ and the total number of tree on the tract $N$, 194 respectively. For the numerator we have:

$195 E\left(\sum_{j=1}^{n} \sum_{i=1}^{N} \frac{A Y_{i}}{V_{i}} Z_{i j}\right)=\sum_{i=1}^{N} \frac{A Y_{i}}{V_{i}} E\left(Z_{i}\right)=\sum_{i=1}^{N} \frac{A Y_{i}}{V_{i}} \frac{V_{i}}{A}=\sum_{i=1}^{N} Y_{i}$

and in the denominator we have: 
$197 E\left(\sum_{j=1}^{n} \sum_{i=1}^{N} \frac{A}{V_{i}} Z_{i j}\right)=\sum_{i=1}^{N} \frac{A}{V_{i}} E\left(Z_{i j}\right)=\sum_{i=1}^{N} \frac{A}{V_{i}} \frac{V_{i}}{A}=N$

198 199

200

201

202

203

204

205

206

207

208

209

210

211

212

213

214

215

216

217

Clearly the ratio between eq. 18 and eq. 19 is the population mean for $Y, \mu_{Y}$. The ratio of means estimator is not unbiased but generally has good properties for a sufficiently large sample size (Cochran, 1977 p. 150-169) when the numerator and denominator of the ratio estimator are unbiased. The difficulty with using this estimator in practical situations is that it is very time consuming to measure $\mathrm{V}$-polygon areas $V_{i}$ for each sample tree in the field.

Because V-polygon areas are difficult to measure we may seek an easily-measured weighting variable that is well correlated with $\mathrm{V}$-polygon area $V_{i}$, though this would not typically result in unbiased estimates of the numerator and denominator of a ratio estimator in equation 17. Technically this would make the numerator in equation 17 a mean of ratios estimator (e.g. Avery and Burkhart 2002 p. 52-53 ) based on mean of ratios $Y_{i} / Z_{i}$ between $Y_{i}$ and a variable $Z_{i}$ the proxy variable for $V_{i}$. One approach to reducing the bias in estimator 6 could be weighting samples inversely proportional to squared sample point-to-plant distance instead of V-polygon area as in equation 17. However, the area of a circle for which the point-to-plant distance is the radius will not generally be equal to the $\mathrm{V}$-polygon area and in all likelihood will be quite different. If we think of this circle as centered around the sample tree or plant we can see that it will very frequently have an area that is smaller than the a "typical" V-polygon area, sometimes (as indicated by Iles 2009) much smaller if the point is close to the sample tree or plant. The area of this circle could also be larger than the V-polygon area if the random plot falls near one of the polygon vertices, depending on how the polygon is shaped. The latter case could be 
218 common for long narrow V-polygons, but this shape is probably not predominant in many

219 forests. In any case, this type of analysis suggests that weighting samples by the inverse of the

220 squared distance from the tree or plant to the random point could be a poor substitute for the V-

221 polygon area $V_{i}$ in equation 17 above. Iles (2009) suggested that the area of the V-polygon could

222 be calculated by averaging the squared length of radii from the selected tree to the V-polygon

223 edge. This method of area computation has been suggested by Matérn (1956) for tree cross-

224 sectional area and Gregoire and Valentine (1995) for planar areas. Iles' (2009) procedure is

225 unbiased for V-polygon area but precise estimation of the area may take an excessive number of

226 measurements in the field. Still, a sample of two or three of the random radii proposed by Iles

227 (2009) might be more stable than use of the more traditional squared point to plant distance, and

228 there would be a smaller chance of obtaining an extremely short distance. For $k$-tree sampling

229 with sufficiently large $k$ Iles (2009) has indicated why the relationship between the higher order

230 V-polygons discussed by Kleinn and Vilkĉo (2006) and the area of the $k$-tree plot defined by the

231 distance from a random point to the $k^{\text {th }}$ closest tree is better for $k>1$ than for $k=1$. Even with $k=2$,

232 Iles (2009) notes this tends to put limits on the minimum length of the radius of the 2-tree plot

233 compare to a 1-tree plot because it has to contain at least two trees. The minimum point-to-plant

234 distance when $k=2$ is half the distance between any two trees sampled by this plan. Clearly the

235 situation tends to get better as $k$ gets larger. There is no lower limit to the radius of a $k=1$-tree

236 plot. Although for a theoretical Poisson distribution there is no limit to how close two trees can

237 be, in actual forests there is always a finite measureable difference between the locations of two

238 trees. Still, situations with clumped distributions can arise in which very small $k$-tree plots can

239 be problematic from a practical point of view. 
241 Figure 2 illustrates the bias in the sample mean obtained from sampling the nearest tree to a

242 random point using equation 13. In Figure 2, the coefficient of variation for $V$ and $Y$ is assumed

243 to be equal so that the results can be plotted on a two dimensional graph. This should suffice for

244 providing a general idea of how large the percent bias can be. Figure 2 indicates that even for

245 low levels of correlation between $Y$ and the $\mathrm{V}$-polygon area $V$ the bias can be large if the

246 coefficient of variation for $Y$ and $V$ is substantial. Widely cited forest measurements books

247 present examples in which error levels of 5\% (Avery and Burkhart 2002 p.39) and 10\%

248 (Bitterlich 1984 p. 141) are used to compute sample sizes. Therefore we conclude that errors of 249 these magnitudes are important in forest sampling and certainly larger errors are to be avoided.

250 While the latter examples actually deal with computing sample size for unbiased estimators, it

251 follows that these levels of bias should be avoided also, all the more so because biases cannot be

252 removed by increasing sample size. Equation 13 indicates that important biases with the

253 methods described above can occur even with low correlations between the individual tree

254 variable $Y_{i}$ and the areas $V_{i}$ of $\mathrm{V}$-polygons if the coefficients of variation for $Y$ and $V$ are high.

255 For example, suppose $\rho_{Y V}=0.2, C_{Y}=0.5$ and $C_{V}=0.5$. Then the bias in the mean of $Y$ expected

256 from sampling the tree or plant nearest to a randomly-located point would be $\rho_{Y V} C_{Y} C_{V} 100=$

$2570.2 \times 0.5 \times 0.5 \times 100=5 \%$.

258 DISCUSSION

259 To the extent that in more mature forest populations we might expect variables such as DBH or

260 basal area to be greater for trees having larger $V_{i}$, then sampling the nearest trees to randomly

261 located points would be a design-biased procedure for any variable correlated with the $V_{i}$. It has

262 been noted that the theory of APA (Avery and Burkhart 2002 p. 331-332) would indicate 
263 correlations between APA and certain tree growth variables commonly occur. In their critique of

264 the "point-centered quarter" method of density estimation, Mark and Esler (1970) found a

265 significant positive relationship between DBH and APA for their data with a slope of 0.648

266 between DBH $(\mathrm{cm})$ and APA $\left(\mathrm{m}^{2}\right)$ where DBH was the independent variable (in the original

267 English units the coefficient was 5.4 with DBH in inches and APA in $\mathrm{ft}^{2}$ ). They apparently did

268 not report an $R^{2}$ or a correlation coefficient for this relationship, however inspection of the

269 sample points surrounding the regression line in the published figure suggest that although

270 significant the correlation was low Figure 2 indicates the levels of bias from a range of values of

271 the correlation coefficient and coefficient of variation. A recent study conducted in a 28 -year-

272 old even-aged coastal redwood (Sequoia sempervirens) forest (Gspalti et al. 2011) found a

273 significant relationship between basal area increment weighted by leaf area (dependent variable)

274 and leaf area divided by weighted APA (independent variable), having an $R^{2}=0.35$ (implying a

275 correlation coefficient $r=0.59$ ). These authors also found high levels of variation in APA due to

276 the characteristic sprouting of redwood. APA for tree $i$ is identical to the area of the V-polygon

277 for tree $i$. This means that to the extent that APA is correlated with individual tree attributes

278 there can be an expectation of bias for several individual tree variables when sample tree are 279 selected as those nearest to randomly-located points in the field.

281 We can also predict some situations in which the bias would be low. In forests that have recently

282 been thinned the correlation between V-polygons and variables such as crown width, basal area

283 and DBH might be reduced because trees have not yet responded to thinning. Plantations

284 planted with very accurate spacing and having little mortality may have low coefficients of

285 variation among the $V_{i}$ areas of $\mathrm{V}$-polygons associated with sample trees, leading to low bias. 
286 However, it is hard to imagine a realistic situation in which the coefficient of variation among V-

287 polygons would be zero because some mortality usually occurs and in actual plantations the

288 spacing is never perfect. The coefficient of variation among V-polygons increases with age even

289 in plantations as gaps arise due to mortality and especially when commonly-prescribed

290 sivicultural treatments such as thinning are applied.

292 It is conceivable for some variables that a negative bias could occur from sampling trees nearest

293 to a randomly-located point. Particularly in uneven-aged forests, PAI (periodic annual

294 increment) for DBH (in the most recent years prior to sampling) may be lower for large trees

295 past the point of culmination of PAI for DBH, and if the V-polygons for such trees tend to be

296 larger there could be a negative correlation between PAI for DBH (e.g. as measured on

297 increment cores) and the areas of V-polygons associated with sample trees. According to

298 equation (13) such a negative correlation would lead to negative bias from sampling trees nearest

299 to a randomly located point in the field. In this situation estimates of DBH increment could be

300 too low. On the other hand in plantations or other even-aged forests, large trees with large V-

301 polygons may have greater PAI than other trees of the same current age, leading to positive

302 biases in PAI for the years just prior to and up to the current year of sampling.

304 There is extensive ongoing research concerning V-polygons due to their importance in many

305 scientific fields. Ferenc and Néda (2007) have recently published an investigation concerning

306 the size distribution of Poisson Voronoi cells that models this size distribution (for which no

307 exact mathematical form is known in 2 or higher dimensions) with a simple formula. Since the 
308 Poisson distribution has sometimes been used to approximate the spatial distribution for

309 randomly-distributed trees or other plants, this type of result may help us gain intuition into the

310 possible magnitude of the coefficient of variation for the areas of V-polygons, which directly

311 impacts the bias formulae discussed above. However many forest stands have clumped spatial

312 distributions and young plantations have uniform spatial distributions that are not adequately

313 modeled by the Poisson distribution.

315 Advances in GPS and/or other technology may make it significantly easier to map some tree

316 locations in the field, making the computation of the areas of V-polygons feasible for some

317 sample trees. At first this might lead to a 2-stage sampling procedure in which V-polygons are

318 measured for a small subset of a larger sample of plants or trees located nearest to a random

319 point on in the field. Possibly these data could be used to estimate the bias using equation 12

320 with estimated values substituted for population values. More likely, ratio or regression

321 estimators could be used to adjust biased estimators from the large sample using paired biased

322 and unbiased estimators in the small sample, reducing bias to more acceptable levels.

\section{CONCLUSIONS}

325 The main conclusion from the study is that the design-bias percentage from using a simple unweighted average to estimate plant attributes when selecting sample trees or other plants

327 nearest to points located randomly and uniformly on a land area has been shown to be equal to

$328 \rho_{Y V} C_{Y} C_{V} 100$. Using this formula it has also been concluded that important biases of greater than 
329

330

331

332

333

334

335

336

337

338

339

340

341

342

343

344

345

346

347

348

349

350

351

$5 \%$ are possible in many realistic forest conditions. These biases are likely to occur because ecological and silvicultural principles lead to the expectation that many tree attributes are significantly influenced by growing space.. If we think that correlations between V-polygon size and some individual tree variables could range from 0.8 to 0.5 and coefficients of variation for these variables and for $\mathrm{V}$-polygons could range from $20 \%$ to $50 \%$, then we can quickly calculate that biases from as low as $2 \%$ to as high as $20 \%$ may be possible from this procedure depending on the characteristics of the forest of interest. As indicated above, even lower levels of correlation between an individual tree variable of interest $Y$ and the area of the associated Vpolygon $V_{i}$ will imply important levels of bias if the coefficients of variation for $Y$ and $V$ are large (Figure 2). A correlation coefficient of only $\rho_{Y V}=0.1$ if combined with coefficients of variation (in terms of proportion) of 0.70 for $Y$ and $V$ would lead to a bias of almost $5 \%$. Although unbiased estimators are possible they depend on measurement of sample V-polygons which with current technology is difficult in the field. Therefore the procedure of selecting sample plants or trees nearest to a randomly located point in the field should be used with caution if it is used at all. This analysis confirms in a more formal way the observations of Clutter et al. (1983 p. 119) concerning the collection of increment cores from the tree closest to a random point location in the field.

\section{ACKNOWLEDGEMENTS}

The author would like to thank Dr. Jeff Gove, Dr. Timothy Gregoire and two anonymous reviewers for valuable comments, and Dr. Steen Magnussen for discussions concerning estimation of per-tree attributes with distance sampling. This article has been approved for publication by the Oklahoma Agricultural Experiment Station and supported by project OKL02843. 


\section{REFERENCES}

353 Avery, T.E., and Burkhart, H.E. 2002. Forest measurements $5^{\text {th }}$ ed. McGraw-Hill, NY. 456p.

355 Barabesi, L. and Fattorini, L.. 1998. The use of replicated plot, line and point sampling for

356 estimating species abundance and ecological diversity. Environ. Ecol. Stat. 5:353-370.

358 Bitterlich, W. 1984. The relascope idea: relative measurements in forestry. Commonwealth 359 Agricultural Bureaux, Farnham Royal, Englnd. 242 p.

Brown, G.S. 1965. Point density in stems per acre. New Zealand Forestry Service Research

362 Notes 38:1-11

363

364 Clutter, J.L., Fortson, J.C., Pienaar, L.V., Brister, G.H., and Bailey, R.L. 1983. Timber 365 management: a quantitative approach. John Wiley and Sons, NY. 333p.

367 Cochran, W.G. 1977. Sampling techniques, third edition. John Wiley, NY. 428p. 
370 Ferenc, J-S. and Néda, N. 2007. On the distribution of Poisson Voronoi cells. Physica A $371 \quad 385: 518-526$.

372

373 Fehrmann, L., Gregoire, T.G., and Kleinn, C. 2012. Triangulation based inclusion probabilities:

374 a design-unbiased sampling approach. Environ. Ecol. Stat. 19:107-123.

375

376 Gove, J.H. and Patil, G.P. 1998. Modeling the basal area-size distribution of forest stands: a 377 compatible approach. For. Sci. 44(2):285-297.

378

379

380 Gregoire, T.G. and H.T. Valentine. 1995. A sampling strategy to estimate the area and 381 perimeter of irregularly-shaped planar regions. For. Sci. 41:470-476.

383 Gspalti, M., Sterba, H. and O'hara, K.L.. 2011. The relationship between available area

384 efficiency and area exploitation index in an even-aged coast redwood (Sequoia sempervirens)

385 stand. Forestry 85: 567-577.

386 
388 Iles, K. 2009. "Nearest-tree" estimations: a discussion of their geometry. International Jor.

389 Math. Comp. Forestry \& Natural Res. 1(2):47-51.

390

391 Kleinn, C. and Vilckko, F. 2006. Design-unbiased estimation for point-to-tree distance sampling.

392 Can. J. For. Res. 36:1407-1414.

393

394 Lappi, J. and Bailey, R.L. 1987. Estimation of diameter increment function or other tree

395 relations using angle-count samples. For. Sci. 33(3):725-739.

396

397 Mark, A.F. and Esler, A.E. 1970. An assessment of the point-centered quarter method of

398 plotless sampling in some New Zealand forests. Proc. New Zealand Ecol. Soc. 17:106-110.

399

400 Matérn, B. 1956. On the geometry of the cross-section of a stem. Medd. Stat. Skogsforkn Inst.

$401 \quad 46(11) .28 p$.

402

403 Mead, R. 1966. A relationship between individual plant-spacing and yield. Annals of Botany

$404 \quad 30: 301-309$.

405 
406 Mercier, F. and Baujard, G. 1997. Voronoi diagrams to model forest dynamics in French 407 Guiana. In: Proceedings of GeoComputation '97 \& SIRC '97 p. 161-171. Second annual 408 conference of GeoComputation '97 \& SIRC '97, University of Otago, New Zealand, 26-29 409 August 1997.

410

411 Overton, W.S. and Stehman, S.V. 1995. The Horvitz-Thompson theorem as a unifying 412 perspective for probability sampling: with examples from natural resource sampling. The 413 American Statistician 49: 261-268.

415 Palley, M.N. and Horwitz, L.G. 1961. Properties of some random and systematic point 416 sampling estimators. For. Sci. 7:52-65.

418 Patil, G.P. and Rao, C.R. 1978. Weighted distributions and size-biased sampling with 419 applications to wildlife populations and human families. Biometrics 34:179-189.

421 R Development Core Team. 2012. R: A Language and Environment for Statistical Computing.

422 R Founda-tion for Statistical Computing, Vienna, Austria. URL http://www.R-project.org. ISBN 423 3-900051-07-0. 
426 Turner, R. 2015. Package 'deldir' version 0.1-9. https://www.stat.auckland.ac.nz/ rolf/ (last

427 accessed 8/1/2015.

428

429 Venables, W.N. and Ripley, B.D. 2002. Modern Applied Statistics with S. Springer, New

430 York, fourth edition. URL http://www.stats.ox.ac.uk/pub/MASS4. ISBN 0-387-95457-0.

431

432

433 Wikepedia authors, 2015. Voronoi diagram. http://en.wikipedia.org/wiki/Voronoi_diagram (last

434 accessed June 5, 2015).

435 
437 Figure 1. A Voronoi tessellation for a typical forested area in which circles represent tree

438 locations and the dashed lines are the boundaries of $\mathrm{V}$-polygons enclosing the trees, with the $\mathrm{x}$ 439 and y axes measured in meters.

440

441 Figure 2. Percent bias in the sample mean of $Y$ versus the coefficient of variation for $Y$ and $\mathrm{V}$ 442 polygon level $V$ assuming that they are equal, for levels of the correlation coefficient $r$ from 0.1 443 to 1 . 


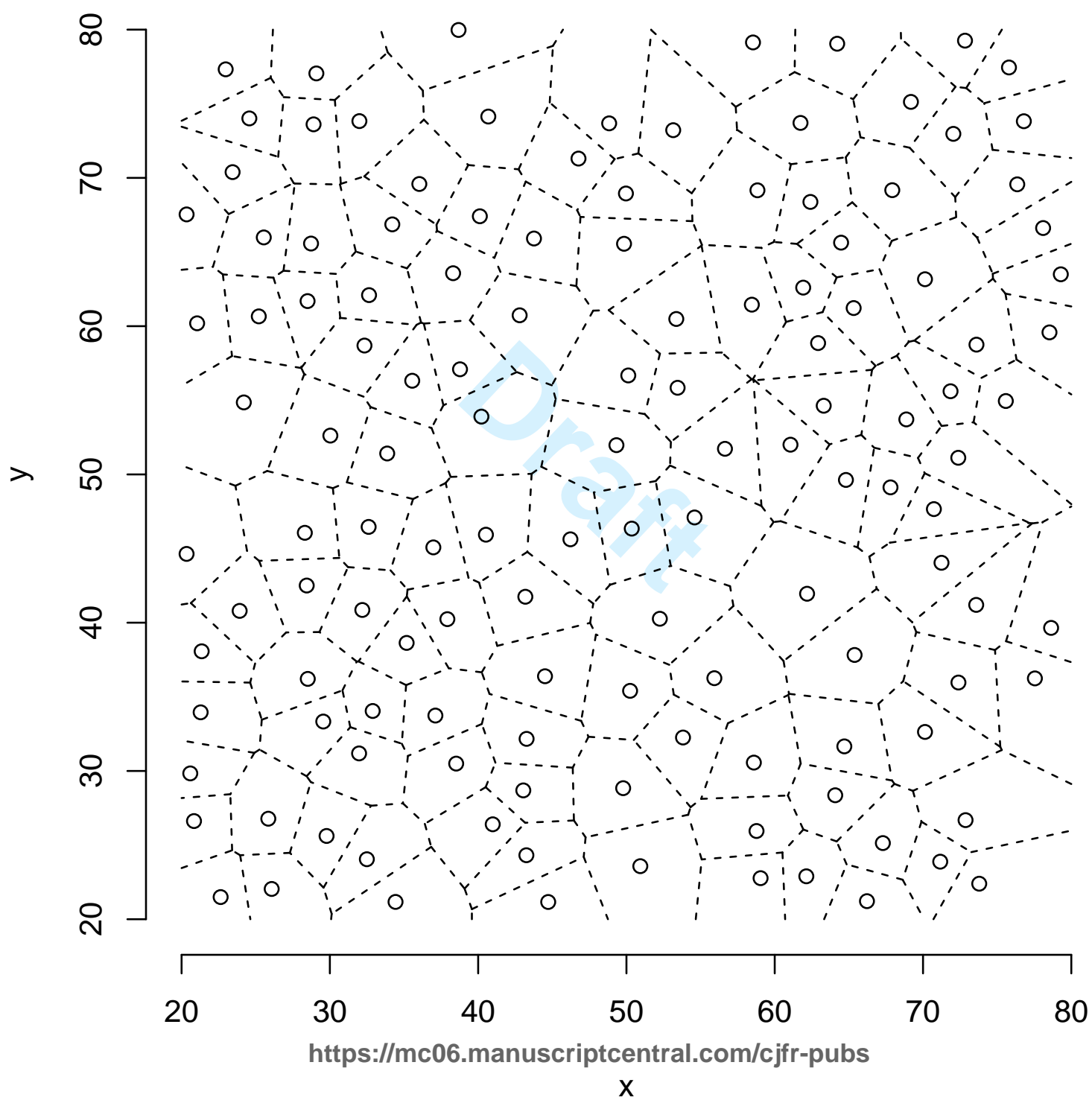




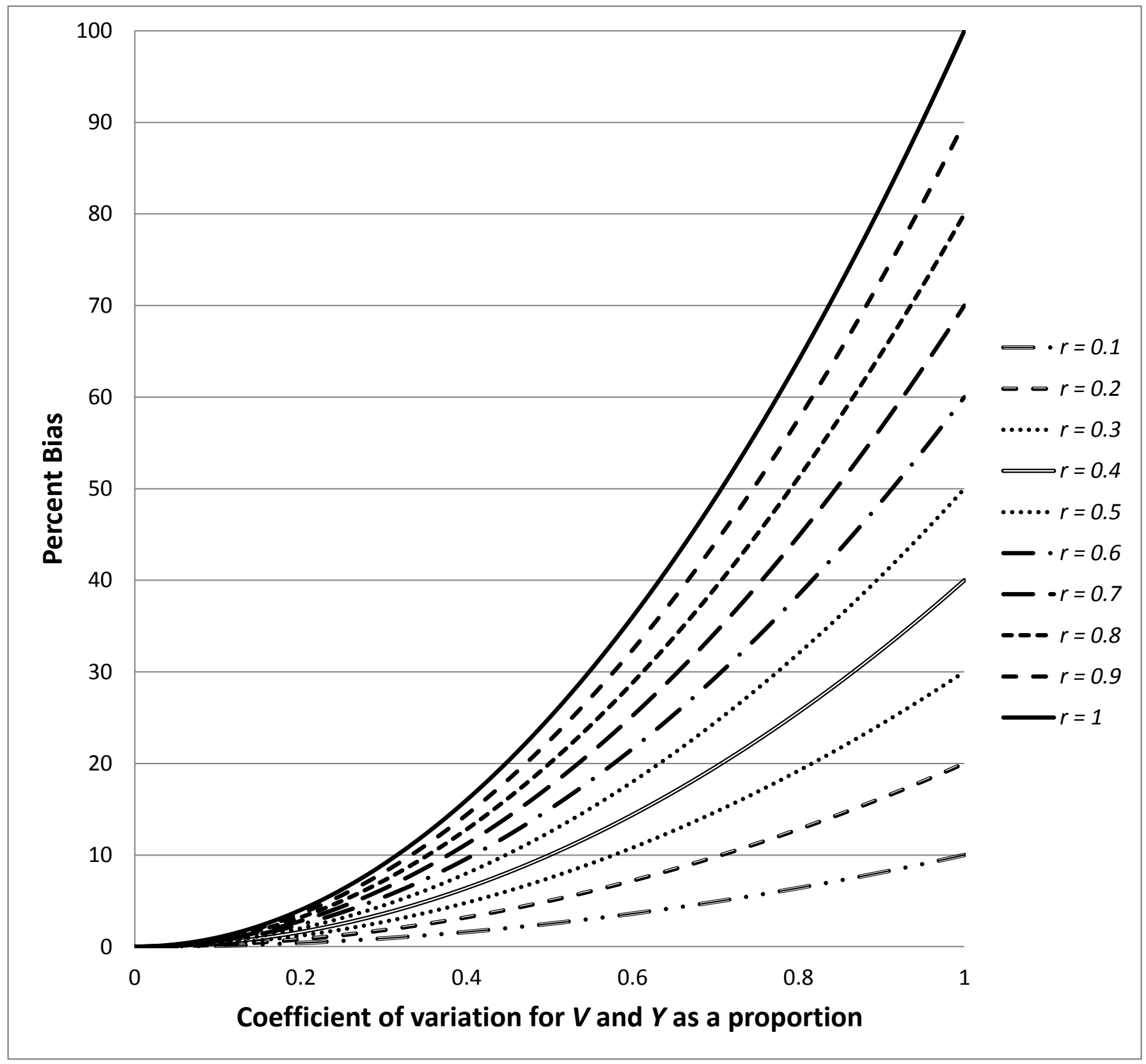

